\title{
Vanishing Lung Syndrome: An Idiopathic Bullous Emphysema Mimicking Pneumothorax
}

\author{
Muhammad N. Yousaf 1, 2, 3, 4, Nim N. Chan 5, 6, 7,8, Adrien Janvier 6, 9 \\ 1. Internal Medicine, MedStar Union Memorial Hospital, Baltimore, USA 2. Internal Medicine, MedStar Franklin Square \\ Medical Center, Baltimore, USA 3. Internal Medicine, MedStar Good Samaritan Hospital, Baltimore, USA 4. Internal \\ Medicine, MedStar Harbor Hospital, Baltimore, USA 5. Medicine, MedStar Union Memorial Hospital, Baltimore, USA 6. \\ Medicine, MedStar Franklin Square Medical Center, Baltimore, USA 7. Medicine, Medstar Good Samaritan Hospital, \\ Baltimore, USA 8. Medicine, MedStar Harbor Hospital, Baltimore, USA 9. Medicine, MedStar Georgetown University, \\ School of Medicine, Washington, DC, USA
}

Corresponding author: Muhammad N. Yousaf, muhammad.n.yousaf@medstar.net

\begin{abstract}
Vanishing lung syndrome (VLS) is also referred to as idiopathic giant bullous emphysema and is a rare manifestation of chronic obstructive pulmonary disease (COPD). Middle-aged tobacco smokers, younger marijuana users, and those with alpha-1-antitrypsin deficiency may especially be affected. The clinical and radiographic findings of VLS may initially be misinterpreted as spontaneous pneumothorax. High-resolution CT is the diagnostic imaging modality of choice in these patients and can help to differentiate VLS from pneumothorax. Such imaging also helps guide appropriate management. Management of VLS ranges from a conservative to a surgical approach depending upon patients' comorbidities and candidacy for surgical resection. We present a case of a 64-year-old man with frequent hospitalizations for COPD exacerbation admitted with worsening shortness of breath and was found to have giant bullae mimicking a pneumothorax on the initial presentation.
\end{abstract}

Received 07/23/2020

Review began $07 / 28 / 2020$ Review ended 08/03/2020 Published 08/06/2020

(c) Copyright 2020

Yousaf et al. This is an open access article distributed under the terms of the Creative Commons Attribution License CC-BY 4.0., which permits unrestricted use, distribution, and reproduction in any medium, provided the original author and source are credited.
Categories: Emergency Medicine, Internal Medicine, Pulmonology

Keywords: vanishing lung syndrome, giant bulla, emphysema, pneumothorax, copd, idiopathic bullous emphysema, lung volume reduction surgery

\section{Introduction}

Vanishing lung syndrome (VLS), also known as idiopathic giant bullous emphysema represents a rare form of irreversible damage to the pulmonary parenchyma often due to chronic obstructive pulmonary disease (COPD). Patients with this condition typically have a long history of smoking or COPD but may also be younger with a history of marijuana use or have alpha-1 antitrypsin deficiency [1-3]. VLS is associated with a spectrum of clinical manifestations, including worsening dyspnea, cough, declining pulmonary function, and occasionally spontaneous pneumothorax due to ruptured bullae [4]. Robert et al. described the radiological criteria for VLS in 1987 as the presence of giant bullous emphysema predominantly involving one or both upper lung lobes and occupying at least one-third of the hemithorax. These bullae compress the surrounding parenchyma of the lower lung lobes or mediastinum [5]. Subpleural bullae with paraseptal emphysema or centrilobular emphysema may also be seen. Idiopathic giant bullous emphysema may mimic the presentation of pneumothorax with worsening dyspnea, hypoxia, and a chest radiograph revealing an absence of pulmonary markings. However, in the case of a true pneumothorax, a white line representing visceral pleura that has separated from the chest wall is often seen. Clinical concern for pneumothorax should be immediately evaluated with an urgent CT scan of the chest. We present a case of a 64-year-old man with a history of smoking and COPD presenting with clinical and radiographic findings of VLS mimicking a spontaneous pneumothorax on presentation.

\section{Case Presentation}

A 64-year-old man with a 50 packs per year smoking history, COPD on three-liter (L) home oxygen, and right lung cancer status post resection 25 years prior to admission presented to the emergency room with worsening shortness of breath, non-productive cough, wheezing, and generalized weakness for the past few weeks. At presentation, vitals were notable for blood pressure of 165/98 mm Hg, tachypnea (RR 22/minute), and oxygen saturation of $92 \%$ on $3 \mathrm{~L}$ of nasal cannula. On physical examination, he was dyspneic, cachectic appearing with diminished breath sounds more on left hemithorax, and bilateral diffuse wheezing. Laboratory workup was unremarkable. Chest radiograph revealed bullous changes involving both upper lobes and particularly the left hemithorax. Mild blunting of the left costophrenic angle from a small left pleural effusion or pleural thickening was also seen (Figure 1). 


\section{Cureus}

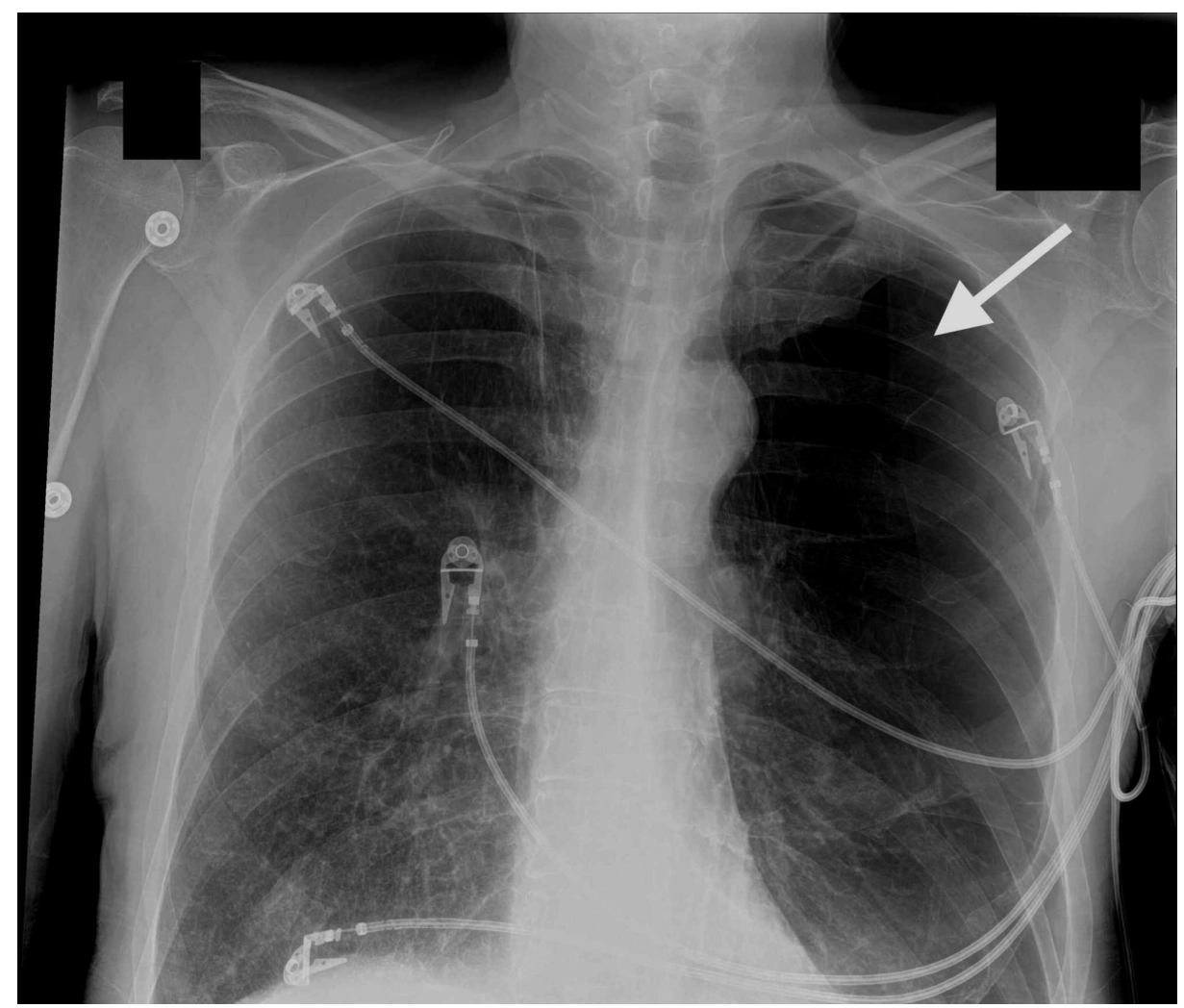

\section{FIGURE 1: Chest radiograph}

Chest radiograph (AP view) showing bullous changes involving bilateral upper lobe with severe bullous emphysematous changes particularly in the left hemithorax.

Given his respiratory distress and radiographic findings concerning for a pneumothorax, a CT of the chest was performed and pneumothorax was ruled out. On CT scan, we noted that giant bullae compressed the left lower lung lobe (Figures 2, 3). These radiological findings were consistent with VLS that had initially mimicked a pneumothorax. Our patient was not deemed a candidate for lung volume reduction surgery. He improved modestly with oral prednisone and scheduled nebulizer treatments. 


\section{Cureus}

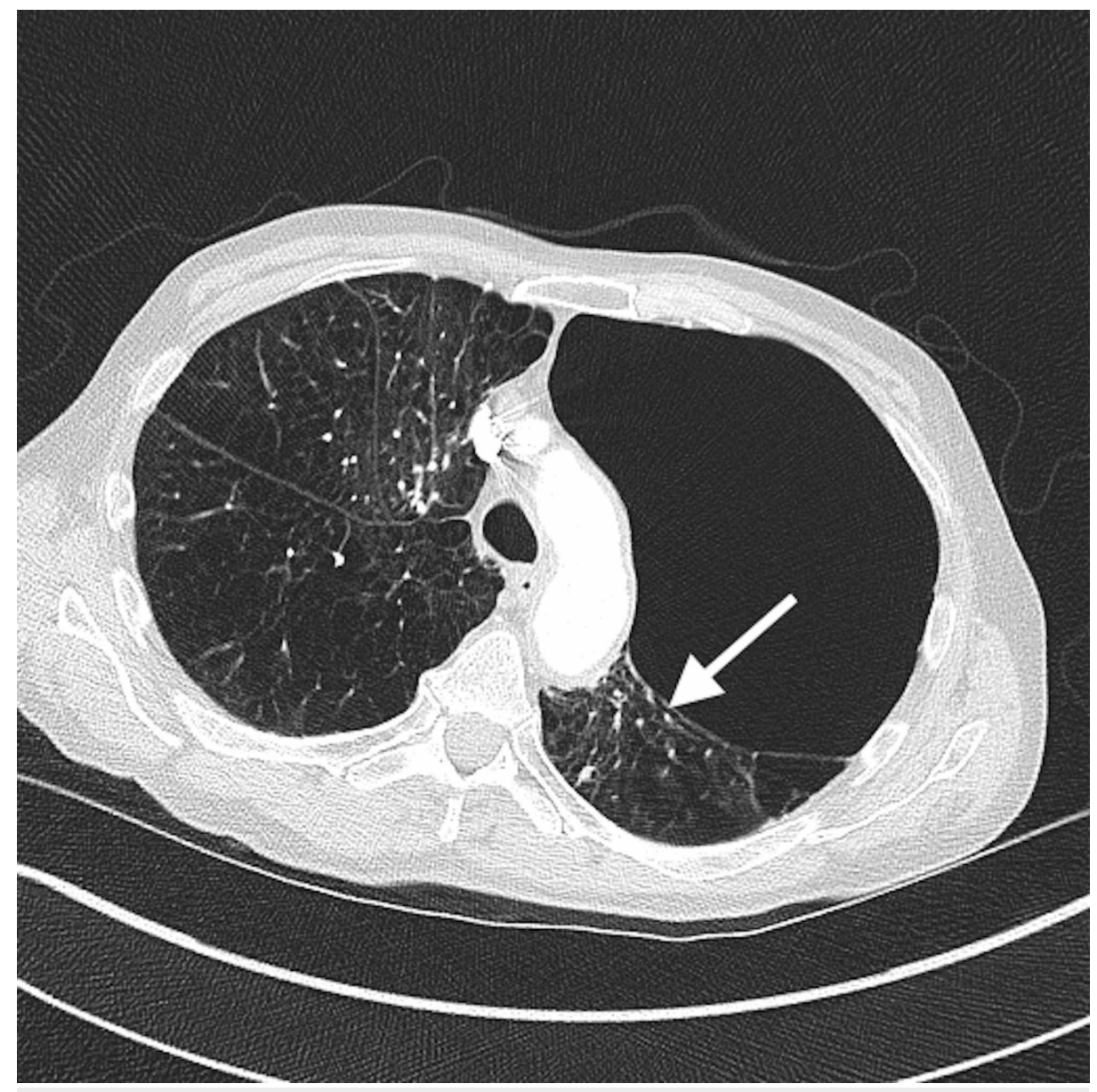

FIGURE 2: CT scan of the chest

Axial section of CT showing severe bilateral emphysematous changes with a giant bulla involving left upper lobe, compressing left lower lobe parenchyma (arrow). 


\section{Cureus}

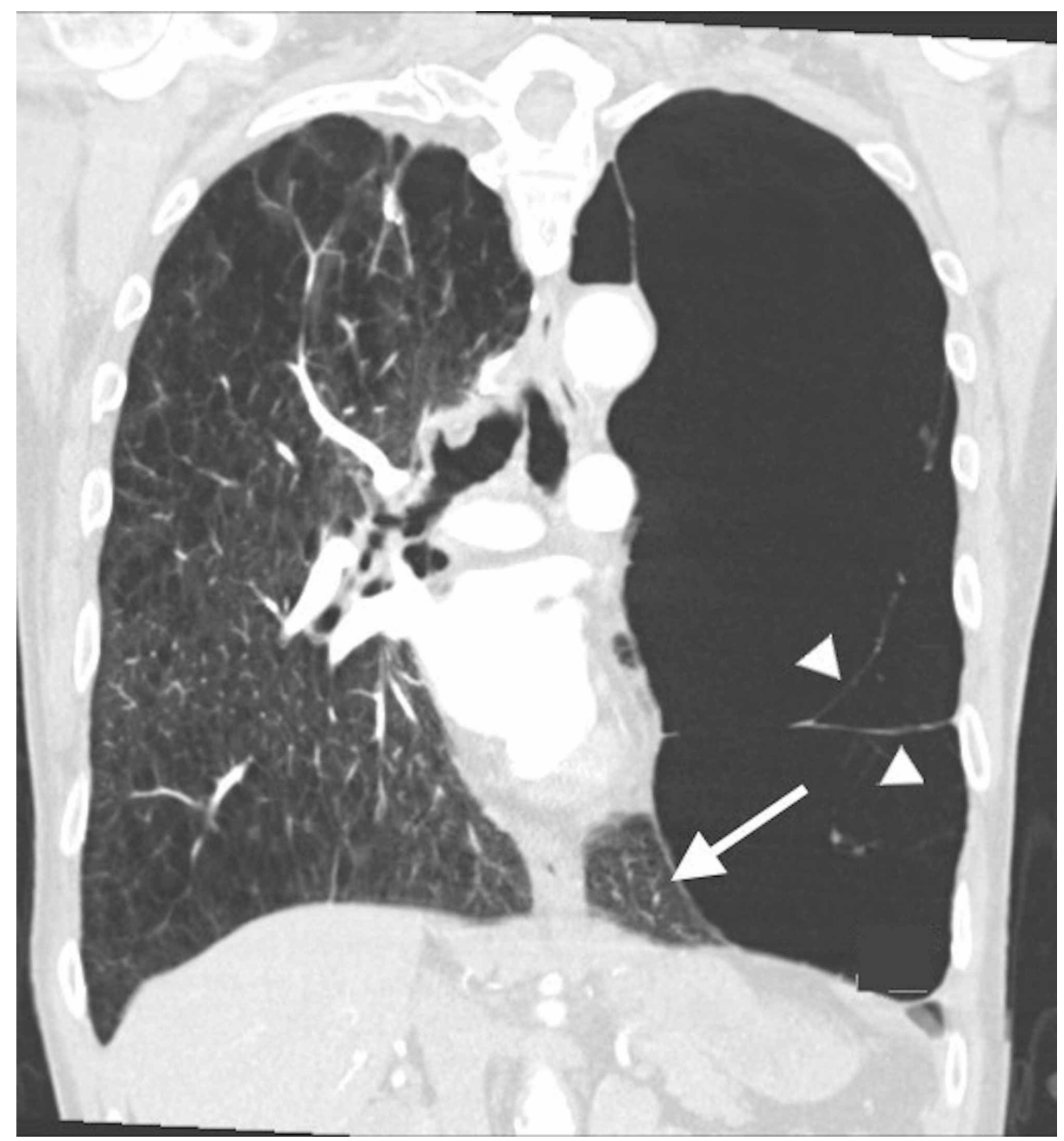

\section{FIGURE 3: CT scan of the chest}

Coronal view showing a giant bulla with septae (arrowheads) involving entire left upper lobe of the lung, occupying more than two-third of the left hemithorax resulting in compression of left lower lobe of lung parenchyma (pointed with arrow).

\section{Discussion}

Emphysema is a common manifestation of COPD due to the destruction of pulmonary parenchyma and permanent alveolar dilation. An estimated $11.4 \%$ of the world's population is affected with COPD, and it is the third leading cause of death in the United States [6-8]. Enzymatic destruction of the alveolar wall from elastase produced by alveolar macrophages and neutrophils plays a crucial role in the pathogenesis of bullae formation. Bullae occurring in the setting of emphysema are by definition air-filled cavities greater than 1 $\mathrm{cm}$ in diameter. They result in poor gas exchange due to smaller total alveolar surface area and fibrosis of the alveolar membrane itself [9]. These changes lead to a ventilation-perfusion mismatch that results in hypoxemia and hypercapnia. In some cases, enlarging bullae cause compression of the surrounding lung parenchyma and mediastinum.

For both symptomatic and asymptomatic patients, workup includes pulmonary function testing and a serum alpha-1-antitrypsin level. However, differentiating a pneumothorax from VLS is important because the management differs. In asymptomatic patients with VLS, a more conservative approach employing nebulized bronchodilators is reasonable. However, in symptomatic patients and those with actual pneumothorax, surgical intervention is usually required. The surgical management of VLS includes stapled bullectomy, endocavitary drainage, volume reduction with video-assisted thoracoscopic surgery, one-way endobronchial valves, or lung transplant. In patients undergoing surgical interventions, improvements in pulmonary function (forced expiratory volume in one second [FEV1] and FEV1/forced vital capacity [FVC] ratio), subjective dyspnea scoring, and quality of life resulted [10]. While there is no improvement in the mortality, however, a decreased risk of infection and pneumothorax is associated with this approach [10]. Our patient was not deemed a candidate for the lung volume reduction surgery and was, therefore, managed conservatively. 


\section{Conclusions}

Differentiating VLS from pneumothorax is essential to determining the best plan of care. Acute respiratory distress with hypoxemia may be the early manifestation of pneumothorax due to ruptured giant bullae, and prompt chest tube placement is critical. In VLS, lung volume reduction surgery improves patients' symptoms, pulmonary function, quality of life, and the need for re-hospitalization. In general, patients should be counseled to stop smoking tobacco and marijuana. Those who are poor candidates for surgical resection may benefit from conservative management or palliation.

\section{Additional Information \\ Disclosures}

Human subjects: Consent was obtained by all participants in this study. Conflicts of interest: In compliance with the ICMJE uniform disclosure form, all authors declare the following: Payment/services info: All authors have declared that no financial support was received from any organization for the submitted work. Financial relationships: All authors have declared that they have no financial relationships at present or within the previous three years with any organizations that might have an interest in the submitted work. Other relationships: All authors have declared that there are no other relationships or activities that could appear to have influenced the submitted work.

\section{References}

1. Hii SW, Tam JD, Thompson BR, Naughton MT: Bullous lung disease due to marijuan . Respirology. 2008, 13:122-127. 10.1111/j.1440-1843.2007.01186.x

2. Hutchison DCS, Cooper D: Alpha-1-antitrypsin deficiency: smoking, decline in lung function and implications for therapeutic trials. Respir Med. 2002, 96:872-880. 10.1053/rmed.2002.1381

3. Beshay M, Kaiser H, Niedhart D, Reymond MA, Schmid RA: Emphysema and secondary pneumothorax in young adults smoking cannabis. Eur J Cardiothorac Surg. 2007, 32:834-838. 10.1016/j.ejcts.2007.07.039

4. Gao X, Wang H, Gou K, et al.: Vanishing lung syndrome in one family: five cases with a 20-year follow-up . Mol Med Rep. 2015, 11:567-570. 10.3892/mmr.2014.2673

5. Roberts L PC, Chen JTT, Goodman LR, Ravin CE: Vanishing lung syndrome: upper lobe bullous pneumopathy. Rev Interam Radiol. 1987, 12:249-255.

6. Lone YA, Dar AM, Sharma ML, et al.: Outcome of the surgical treatment of bullous lung disease: a prospective study. Tanaffos. 2012, 11:27-33.

7. Sharma N, Justaniah AM, Kanne JP, Gurney JW, Mohammed TL: Vanishing lung syndrome (giant bullous emphysema): CT findings in 7 patients and a literature review. J Thorac Imaging. 2009, 24:227-230. 10.1097/RTI.0b013e31819b9f2a

8. Adeloye D, Chua S, Lee C, et al.: Global and regional estimates of COPD prevalence: systematic review and meta-analysis. J Glob Health. 2015, 5:020415.

9. Im Y, Farooqi S, Mora A Jr: Vanishing lung syndrome. Proc (Bayl Univ Med Cent). 2016, 29:399-401. 10.1080/08998280.2016.11929486

10. Palla A, Desideri M, Rossi G, Bardi G, Mazzantini D, Mussi A, Giuntini C: Elective surgery for giant bullous emphysema: a 5-year clinical and functional follow-up. Chest. 2005, 128:2043-2050.

10.1378/chest.128.4.2043 